\title{
Contralateral Lobe Volume Brings Us One Step Closer to the Prediction of Hypothyroidism Following Partial Thyroid Resection
}

\author{
Lindsay E. Kuo, MD, MBA ${ }^{1}$, Douglas L. Fraker, $\mathrm{MD}^{2}$, and Rachel R. Kelz, MD, MSCE ${ }^{3}$ \\ ${ }^{1}$ Department of Surgery, Center for Surgery and Health Economics, University of Pennsylvania, Philadelphia, PA; \\ ${ }^{2}$ Department of Surgery, Division of Endocrine and Oncologic Surgery, University of Pennsylvania, Philadelphia, PA; \\ ${ }^{3}$ Department of Surgery, Division of Endocrine and Oncologic Surgery, Center for Surgery and Health Economics, \\ University of Pennsylvania, Philadelphia, PA
}

We live in an age of patient empowerment in which patients can search online for information about their procedures, and expect (rightfully so) to have informed discussions about the risks and benefits of an operation with their surgeon. Consequently, surgeons must be prepared to have thorough conversations with patients about the pros and cons of an operation. Extensive work has been done to determine the optimal operation for patients with thyroid nodules: the Bethesda criteria laid the groundwork for determining the best operation based on thyroid nodule cytopathology, ${ }^{1}$ and testing with AFIRMA or for genetic mutations has furthered this field.

Patients can choose to undergo a partial thyroid resection or a complete thyroidectomy for lesions diagnosed as follicular neoplasms, suspicious for follicular neoplasms, or suspicious for malignancy. The risks associated with partial resection are less severe than those of complete thyroidectomy. However, the risks of both operations are low and therefore the decision is usually centered on the patient's willingness to consider a second surgery, if necessary, and the uncertain potential for needing long-term thyroid hormone replacement. Data to guide patient decision making on the likelihood of requiring a thyroid hormone supplement have been limited to date. Reports of thyroid hormone replacement intolerance due to an

(C) Society of Surgical Oncology 2017

First Received: 30 November 2016;

Published Online: 27 March 2017

R. R. Kelz, MD, MSCE

e-mail: Rachel.Kelz@uphs.upenn.edu impaired sense of well-being, fatigue, weight gain, hair loss, nightmares and other untoward complications of treatment have marred patient perception of the desirability of thyroid hormone replacement.

In a prospective study, Lang et al. cleverly examined the association between measurements of the contralateral thyroid lobe volume from the preoperative ultrasound and the development of biochemical hypothyroidism following hemithyroidectomy. ${ }^{2}$ A risk prediction scoring system was developed to prognosticate the likelihood of developing postoperative hypothyroidism; this model incorporated body surface area (BSA) - adjusted remnant volume, the preoperative thyroid-stimulating hormone (TSH) level, and the number of nodules found in the resected lobe. ${ }^{2}$ These findings strengthen the observations by De Carlucci et al. regarding the importance of remnant volume, ${ }^{3}$ and are concordant with the existing body of literature that has demonstrated the presence of antithyroid peroxidase antibodies, thyroiditis, a multinodular goiter, preoperative thyrotoxicosis, and a TSH level within the high-normal range to be associated with the development of biochemical hypothyroidism following hemithyroidectomy. ${ }^{3-7}$

Despite the novel approach to risk-stratify patients regarding the development of biochemical hypothyroidism, the paper does not solve the problem of predicting clinically significant hypothyroidism (i.e. hypothyroidism requiring treatment) as most postoperative biochemical hypothyroidism resolves spontaneously. ${ }^{6}$ In this study, 17 of 44 (38.6\%) patients with postoperative biochemical hypothyroidism eventually required thyroxine supplementation. This result fits in the larger published range of $1.7^{5}-68.8 \%^{4}$; the wide range can be attributed to differences in patient 
population, duration of postoperative follow-up, and definition of hypothyroidism. A large meta-analysis determined an approximate risk of biochemical hypothyroidism of $20 \%$, and, perhaps more important to patients, a risk of just $4 \%$ for developing clinical hypothyroidism. ${ }^{6}$

Given these wide ranges for the risk of developing biochemical hypothyroidism, and the overall low risk of developing hypothyroidism requiring treatment, how does one best counsel patients preoperatively and treat patients postoperatively? Addressing the latter question is perhaps more straightforward. Given the low rate of clinical hypothyroidism, patients should not be empirically started on thyroxine treatment postoperatively. Instead, regular thyroid function tests may be considered to identify patients with biochemical hypothyroidism; ${ }^{4-6,8}$ however, the practicality and cost effectiveness of this solution is unclear, particularly in light of the low rate of overt postoperative hypothyroidism. Incorporating a risk prediction score such as the one put forth in this study by Lang et al. may be a useful way to selectively screen high-risk individuals postoperatively, rather than testing all patients, or to test more frequently or for a longer duration postoperatively than for all-comers.

Furthermore, how does one counsel a patient preoperatively about his or her risk of postoperative hypothyroidism? While more information can be a powerful tool (the incorporation of thyroid remnant volume as determined by preoperative ultrasound is one such example), effective deployment of this information is another step entirely. As studies have failed to identify factors predictive of clinical hypothyroidism, new information on the risk of biochemical hypothyroidism must be shared, along with details of its limitations. Providing an individualized risk prediction for biochemical hypothyroidism following hemithyroidectomy, along with facts on the limitations of the estimate, will undoubtedly facilitate improved informed consent and shared decision making. Optimal information on the risk of clinically significant hypothyroidism for patients is still just out of reach, but we are one step closer with the addition of contralateral lobe volume to the equation.

\section{REFERENCES}

1. Baloch ZW, LiVolsi VA, Asa SL, et al. Diagnostic terminology and morphologic criteria for cytologic diagnosis of thyroid lesions: a synopsis of the National Cancer Institute Thyroid Fine-Needle Aspiration State of the Science Conference. Diagn Cytopathol. 2008;36:425-47.

2. Lang B, Wong C, Wong K, et al. Effect of thyroid remnant volume on the risk of hypothyroidism following hemithyroidectomy: a prospective study. Ann Surg. Oncol. Epub 5 Jan 2017.

3. De Carlucci D Jr., Tavares MR, Obara MT, et al. Thyroid function after unilateral total lobectomy: risk factors for postoperative hypothyroidism. Arch Otolaryngol Head Neck Surg. 2008;134(10):1076-79.

4. Su SY, Grodski S, Serpell JW. Hypothyroidism following hemithyroidectomy: a retrospective review. Ann Surg. 2009;250(6):991-94.

5. Ahn D, Sohn JH, Jeon JH. Hypothyroidism following hemithyroidectomy: incidence, risk factors, and clinical characteristics. $J$ Clin Endocrinol Metab. 2016;101(4):1429-36.

6. Verloop H, Louwerens M, Schoones JW, et al. Risk of hypothyroidism following hemithyroidectomy; systematic review and meta-analysis of prognostic studies. J Clin Endocrinol Metab. 2012;97(7):2243-55.

7. Stoll SJ, Pitt SC, Liu J, et al. Thyroid hormone replacement after thyroid lobectomy. Surgery. 2009;146:554-60.

8. Johner A, Griffith OL, Walker B, et al. Detection and management of hypothyroidism following thyroid lobectomy: evaluation of a clinical algorithm. Ann Surg Oncol. 2011;18:2548-54. 